\title{
AUTHOR INDEX Volume 2
}

Bal, G., Papanicolaou, G. \& Ryzhik, L., Self-averaging in time reversal for the parabolic wave equation

Baxendale, P.H., Lyapunov exponents and resonance for small periodic and random perturbations of a conservative linear system

Berglund, N. \& Gentz, B., Metastability in simple climate models: Pathwise analysis of slowly driven Langevin equations

Blanchard, Ph., see Volchenkov

Bonaccorsi, S., Onsager-Machlup functional for volterra equations perturbed by noise

Cong, N.D. \& Siegmund, S., Dichotomy spectrum of nonautonomous linear stochastic differential equations

Cosenza, M.G., see Volchenkov

Daumail, L. \& Florchinger, P., A constructive extension of Artstein's theorem to the stochastic context

Drakopoulos, V., Kakos, A. \& Nikolaou, N., A probabilistic power domain algorithm for fractal image decoding

Duan, J., Gao, H. \& Schmalfuß, B., Stochastic dynamics of a coupled atmosphere-ocean model

Duncan, T.E., Maslowski, B. \& Pasik-Duncan, B., Fractional Brownian motion and stochastic equations in Hilbert spaces

Egger, J., Master equations for Himalayan valley winds

Farrell, B.F. \& Ioannou, P.J., Optimal perturbation of uncertain systems

Florchinger, P., see Daumail

Fraedrich, K., Fickian diffusion and Newtonian cooling: A concept for noise induced climate variability with long-term memory?

2(2002) 507

2(2002) 49

2(2002) 327

2(2002) 203

2(2002) 587

2(2002) 175

2(2002) 203

2(2002) 251

2(2002) 161

2(2002) 357

2(2002) 225

2(2002) 381

2(2002) 395

2(2002) 251

2(2002) 403

2(2002) 357

2(2002) 327

Gentz, B., see Berglund
Herrmann, S. \& Imkeller, P., Barrier crossings characterize stochastic resonance

2(2002) 413

Imkeller, P. \& Milstein, G.N., Moment Lyapunov exponent for conservative systems with small periodic and random perturbations

2(2002) 25

Imkeller, P. \& Lederer, C., The cohomology of stochastic and random differential equations, and local linearization of stochastic flows

2(2002) 131

Imkeller, P. \& Monahan, A.H., Conceptual stochastic climate models

2(2002) 311

Imkeller, P., see Herrmann

2(2002) 413

Imkeller, P. \& Pavlyukevich, I., Model reduction and stochastic resonance

2(2002) 463

Ioannou, P.J., see Farrell

2(2002) 395

Johnson, R.A., Kloeden, P.E. \& Pavani, R., Two-step transition in nonautonomous bifurcations: An explantion

Kakos, A., see Drakopoulos

Kloeden, P.E., see Johnson

Kupferman, R., Stuart, A.M., Terry, J.R. \& Tupper, P.F., Longterm behaviour of large mechanical systems with random initial data

Lederer, C., see Imkeller

Liao, M., Dynamical properties of Lévy processes in Lie groups

Lopes, A.O. \& Lopes, S.R.C., Convergence in distribution of the periodogram of chaotic processes

Lopes, S.R.C., see Lopes

Maslowski, B., see Duncan

Milstein, G.N., see Imkeller

Milstein, G.N., The asymptotic behavior of semi-invariants for linear stochastic systems

Monahan, A.H., see Imkeller

Monahan, A.H., Correlation effects in a simple stochastic model of the thermohaline circulation

2(2002) 437 
Nakaishi, K., Exponentially strong convergence of nonclassical multidimensional continued fraction algorithms

Nikolaou, N., see Drakopoulos

Papanicolaou, G., see Bal

Pasik-Duncan, B., see Duncan

Pavani, R., see Johnson

Pavlyukevich, I., see Imkeller

Ruffino, P.R.C., Decomposition of stochastic flows and rotation matrix

2(2002) 563

2(2002) 161

2(2002) 507

2(2002) 225

2(2002) 67

2(2002) 463

2(2002) 93

2(2002) 507

2(2002) 357

2(2002) 203

2(2002) 175

Sequeira, S., see Volchenkov

Siegmund, S., see Cong

Sigurgeirsson, H. \& Stuart, A.M., Inertial particles in a random field
Sirvent, V.F., Spectra of recurrence dimension for adic systems

2(2002) 599

Stuart, A.M., see Sigurgeirsson 2(2002) 295

Stuart, A.M., see Kupferman 2(2002) 533

Terry, J.R., see Kupferman 2(2002) 533

Tupper, P.F., see Kupferman 2(2002) 533

Volchenkov, D., Sequeira, S., Blanchard, Ph. \& Cosenza, M.G., Transitions to intermittency and collective behavior in randomly coupled map networks

2(2002) 203

Woyczyński, W.A., see Zirbel

Zähle, M., Long range dependence, no arbitrage and the Black-Scholes formula

2(2002) 109

2(2002) 265

Zirbel, C.L. \& Woyczyński, W.A., Rotation of particles in polarized Brownian flows 